\title{
Typ-2-Diabetes
}

\section{Insulin aspart schrittweise oder sofort drei Boli?}

_ Das optimale Vorgehen, wenn bei einem Typ-2-Diabetiker eine Intensivierung der Insulintherapie erforderlich wird, sollte die randomisierte Studie STEPwise klären. Untersucht wurden die Effekte der klassichen Basal-BolusTherapie (ein Bolus zu jeder Mahlzeit) gegenüber der Gabe eines kurzwirksa-

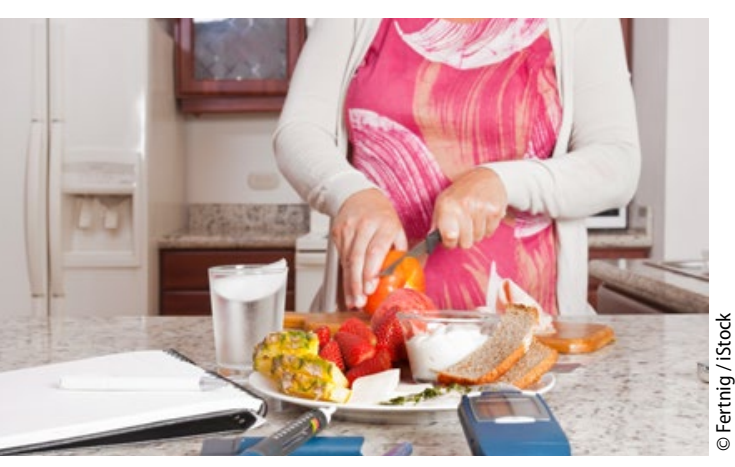

Wie sollten Mahlzeiten insulintechnisch begleitet werden? men Insulins zunächst nur zu einer Mahlzeit.

Die beiden Strategien waren im Hinblick auf die Senkung des $\mathrm{HbA}_{1 \mathrm{c}}$-Wertes und die Hypoglykämierate bei 401 Typ2-Diabetikern miteinander verglichen worden. Als Basalinsulin erhielten alle Patienten Insulin detemir (Levemir ${ }^{\circledast}$ ) und als kurzwirksames Insulin wurde Insulin aspart $\left(\right.$ NovoRapid $\left.^{\circledast}\right)$ gewählt.

Am Ende der Studie nach 32 Wochen wurde in beiden Gruppen eine vergleichbare $\mathrm{HbA}_{1 \mathrm{c}}$-Senkung (-1,12 Prozentpunkte in der STEPwise-Gruppe vs. $-0,98$ Prozentpunkte in der STEPfull-Gruppe) dokumentiert. Ähnlich verhielt es sich mit dem Anteil an Patienten, die den $\mathrm{HbA}_{1 \mathrm{c}}$ - Zielwert $<7 \%$ erreichen konnten (63,3\% vs. $55,9 \%)$.

Der direkte Beginn mit drei Boli des kurzwirksamen Insulins führte jedoch etwas schneller zur $\mathrm{HbA}_{1 \mathrm{c}}$-Reduktion und reduzierte die Blutzuckeranstiege nach allen drei Hauptmahlzeiten. Die schrittweise Intensivierung ging andererseits mit einer geringeren Hypoglykämierate einher. Insbesondere zu Beginn der Therapie mit drei Boli kam es zu einem raschen Anstieg der Hypoglykämierate, die jedoch im weiteren Verlauf wieder abnahm. Die Zufriedenheit der Patienten war mit der stufenweisen Therapie größer, was auch dazu führte, dass weniger Patienten die Studie abbrachen.

Zusammengefasst sprechen die Daten dieser Studie dafür, dass eine schrittweise Intensivierung der Insulintherapie den Patienten eine bessere Möglichkeit bietet, sich mit der prandialen Gabe von Insulin vertraut $\mathrm{zu}$ machen, wodurch Hypoglykämien vermieden werden. Darunter leidet die Qualität der Stoffwechseleinstellung nicht.

Dr. Peter Stiefelhagen

- Nach Informationen von Novo Nordisk

\section{Systolische Herzinsuffizienz}

\section{Kurz notiert}

Kasuistik: multimorbider Patient mit chronisch obstruktiver Lungenerkrankung $\rightarrow$ Bei einem 64-jährigen, adipösen Patienten mit arterieller Hypertonie, schlecht eingestelltem Diabetes mellitus Typ 2, Vorhofflimmern und einer seit 2005 bekannten chronisch obstruktiven Lungenerkrankung (COPD), der wegen Luftnot in die Praxis kommt, steht zunächst die Behandlung der akuten Dyspnoe im Vordergrund, betonte Dr. Peter Kardos, Frankfurt a. Main. Der Patient sei bei einem $\mathrm{FEV}_{1}$-Wert von 40,6\% mit der bisherigen Therapie (Aclidiniumbromid $400 \mu \mathrm{g}$ ) nicht gut eingestellt. Nach Ausschluss einer kardialen Ursache der Atemnot sollte er deshalb möglichst rasch auf eine duale Bronchodilatation umgestellt werden, so Kardos, z. B. auf Aclidinium/Formoterol $340 \mu \mathrm{g} / 12 \mu \mathrm{g}$ (Duaklir ${ }^{\circledR}$ Genuair ${ }^{\oplus}$ ) zweimal täglich. Damit sei eine Zunahme des $\mathrm{FEV}_{1}$ um ca. $100 \mathrm{ml}$ zu erwarten.

Dagmar Jäger-Becker

- Falldiskussion: „Der multimorbide Patient in der Praxis: In terdisziplinäre Diskussion von Kasuistiken zur Priorisierung und Management von Komorbiditäten", 122. Kongress der Deutschen Gesellschaft für Innere Medizin; Mannheim, April 2016 (Veranstalter: AstraZeneca)

\section{Neue Therapieoption ARNI}

_ In der Therapie der Herzinsuffizienz gibt es neue Optionen. Prof. Gregor Simonis, Dresden, sieht durch den kürzlich zugelassenen Angiotensin-Rezeptor-Neprilysin-Inhibitor (ARNI) Sacubitril/Valsartan (Entresto ${ }^{\circ}$ ) eine gesicherte Prognoseverbesserung.

Der supramolekulare Salzkomplex hemmt den schädlichen Einfluss von Angiotensin II und fördert zusätzlich die protektive Wirkung vasoaktiver Peptide. Dieser Ansatz ist der alleinigen Angiotensin-II-Inhibition prognostisch überlegen, wie die bisher größte Langzeitstudie bei systolischer Herzinsuffizienz PARADIGM-HF gezeigt hat. Die Studienpatienten hatten eine eingeschränkte Pumpfunktion $(\mathrm{EF}<35 \%)$, erhöhte BNP-Werte und waren stabil auf ACEHemmer/Angiotensin-Rezeptorblocker und Betablocker eingestellt.
$2 \times 200 \mathrm{mg}$ Sacubitril/Valsartan reduzierten gegenüber $2 \times 10 \mathrm{mg}$ Enalapril das relative Risiko für Tod oder Einweisung wegen Herzinsuffizienz um 20\%. Die Gesamtmortalität sank um $16 \%$.

„In den letzten 25 Jahren hat sich die Herzinsuffizienz-Letalität schrittweise reduziert, der ARNI ist der nächste Schritt auf diesem Weg", kommentierte Simonis. Die Verträglichkeit war gut. 449 (ARNI) versus 516 Patienten (Enalapril) brachen die Therapie nebenwirkungsbedingt ab. $\mathrm{Zu}$ achten sei auf $\mathrm{Hy}$ potonie, so Simonis. Das Medikament muss langsam auftitriert werden. Nur für die Zieldosis ist die Prognoseverbesserung gesichert, so Simonis.

\section{Dr. Dirk Einecke}

- Industriesymposium „Das schwache Herz - Was bedeutet Herzinsuffizienz im Jahr 2016?", 122. Kongress der Deutschen Gesellschaft für Innere Medizin; Mannheim, April 2016 (Veranstalter: Novartis) 